Jacques Blanc-Talon - Cosimo Distante Wilfried Philips · Dan Popescu Paul Scheunders (Eds.)

\title{
Advanced Concepts for Intelligent Vision Systems
}

17th International Conference, ACIVS 2016 Lecce, Italy, October 24-27, 2016 Proceedings

Springer 


\section{Lecture Notes in Computer Science}

Commenced Publication in 1973

Founding and Former Series Editors:

Gerhard Goos, Juris Hartmanis, and Jan van Leeuwen

\section{Editorial Board}

David Hutchison

Lancaster University, Lancaster, UK

Takeo Kanade

Carnegie Mellon University, Pittsburgh, PA, USA

Josef Kittler

University of Surrey, Guildford, UK

Jon M. Kleinberg

Cornell University, Ithaca, NY, USA

Friedemann Mattern

ETH Zurich, Zurich, Switzerland

John C. Mitchell

Stanford University, Stanford, CA, USA

Moni Naor

Weizmann Institute of Science, Rehovot, Israel

C. Pandu Rangan

Indian Institute of Technology, Madras, India

Bernhard Steffen

TU Dortmund University, Dortmund, Germany

Demetri Terzopoulos

University of California, Los Angeles, CA, USA

Doug Tygar

University of California, Berkeley, CA, USA

Gerhard Weikum

Max Planck Institute for Informatics, Saarbrücken, Germany 
More information about this series at http://www.springer.com/series/7412 
Jacques Blanc-Talon · Cosimo Distante

Wilfried Philips · Dan Popescu

Paul Scheunders (Eds.)

\section{Advanced Concepts for Intelligent Vision Systems}

17th International Conference, ACIVS 2016

Lecce, Italy, October 24-27, 2016

Proceedings

iㅡㄹ Springer 
Editors

Jacques Blanc-Talon

DGA

Paris

France

Cosimo Distante

University of Salento

Lecce

Italy

Wilfried Philips

Ghent University

Ghent

Belgium
Dan Popescu

CSIRO, Data 61

Canberra, ACT

Australia

Paul Scheunders

University of Antwerp

Wilrijk

Belgium

ISSN 0302-9743

ISSN 1611-3349 (electronic)

Lecture Notes in Computer Science

ISBN 978-3-319-48679-6

DOI 10.1007/978-3-319-48680-2

Library of Congress Control Number: 2016954946

LNCS Sublibrary: SL6 - Image Processing, Computer Vision, Pattern Recognition, and Graphics

(C) Springer International Publishing AG 2016

This work is subject to copyright. All rights are reserved by the Publisher, whether the whole or part of the material is concerned, specifically the rights of translation, reprinting, reuse of illustrations, recitation, broadcasting, reproduction on microfilms or in any other physical way, and transmission or information storage and retrieval, electronic adaptation, computer software, or by similar or dissimilar methodology now known or hereafter developed.

The use of general descriptive names, registered names, trademarks, service marks, etc. in this publication does not imply, even in the absence of a specific statement, that such names are exempt from the relevant protective laws and regulations and therefore free for general use.

The publisher, the authors and the editors are safe to assume that the advice and information in this book are believed to be true and accurate at the date of publication. Neither the publisher nor the authors or the editors give a warranty, express or implied, with respect to the material contained herein or for any errors or omissions that may have been made.

Printed on acid-free paper

This Springer imprint is published by Springer Nature

The registered company is Springer International Publishing AG

The registered company address is: Gewerbestrasse 11, 6330 Cham, Switzerland 


\section{Preface}

These proceedings gather the selected papers of the Advanced Concepts for Intelligent Vision Systems (ACIVS) conference which was held in Lecce, Italy, during October 24-27, 2016.

This event was the 17th ACIVS. Since the first event in Germany in 1999, ACIVS has become a larger and independent scientific conference. However, the seminal distinctive governance rules have been maintained:

- To update the conference scope on a yearly basis. While keeping a technical backbone (the classic low-level image processing techniques), we have introduced topics of interest such as - chronologically - image and video compression, 3D, security and forensics, and evaluation methodologies, in order to fit the conference scope to our scientific community's needs. In addition, speakers usually give invited talks on hot issues.

- To remain a single-track conference in order to promote scientific exchanges among the audience.

- To grant oral presentations a duration of 25 minutes and published papers a length of 12 pages, which is significantly different from most other conferences.

The second and third items entail a complex management of the conference; in particular, the number of time slots is rather small. Although the selection between the two presentation formats is primarily determined by the need to compose a well-balanced program, papers presented during plenary and poster sessions enjoy the same importance and publication format.

The first item is strengthened by the notoriety of ACIVS, which has been growing over the years: official Springer records show a cumulated number of downloads on July 1, 2016, of more than 440,000 (for ACIVS 2005-2015 only).

The regular sessions also included a couple of invited talks by Professor Andrea Cavallaro (Queen Mary University of London, UK), Professor Thomas B. Moeslund (Aalborg University, Denmark), Professor François Brémond (Inria, France), and Professor Sebastiano Battiato (Catania University, Italy). We would like to thank all of them for enhancing the technical program with their presentations.

ACIVS attracted submissions from many different countries, mostly from Europe, but also from the rest of the world: Algeria, Australia, Austria, Brazil, Belgium, Canada, China, Cyprus, Czech Republic, Denmark, Ecuador, France, Finland, Germany, Hungary, India, Israel, Italy, Korea, Mexico, The Netherlands, Poland, Romania, Russia, Switzerland, Taiwan, Tunisia, Turkey, the Ukraine, United Arab Emirates, the UK, and the USA.

From 137 submissions, 36 were selected for oral presentation and 28 as posters. The paper submission and review procedure was carried out electronically and a minimum of three reviewers were assigned to each paper. A large and energetic Program Committee (87 people), helped by additional reviewers (95 people in total), as listed on the following pages, completed the long and demanding reviewing process. We would like to thank all 
of them for their timely and high-quality reviews, achieved in quite a short time and during the summer holidays.

Also, we would like to thank our sponsors (in alphabetical order) Antwerp University, Commonwealth Scientific and Industrial Research Organization (CSIRO), Ghent University, Institute of Applied Sciences and Intelligent Systems (ISASI), and the National Research Council (CNR) of Italy for their valuable support.

Finally, we would like to thank all the participants who trusted in our ability to organize this conference for the 17th time. We hope they attended a different and stimulating scientific event and that they enjoyed the atmosphere of the ACIVS social events in the city of Lecce.

As mentioned, a conference like ACIVS would not be feasible without the concerted effort of many people and the support of various institutions. We are indebted to the local organizers Marco Leo, Pier Luigi Mazzeo, Paolo Spagnolo, Pierluigi Carcagní, and Marco Del Coco, for having smoothed all the harsh practical details of an event venue, and we hope to welcome them in the near future.

July 2016

Jacques Blanc-Talon Cosimo Distante Wilfried Philips Dan Popescu Paul Scheunders 


\title{
Organization
}

Acivs 2016 was organized by the University of Salento, located in Lecce, Italy.

\section{Steering Committee}

\author{
Jacques Blanc-Talon \\ Cosimo Distante \\ Wilfried Philips \\ Dan Popescu \\ Paul Scheunders
}

\author{
DGA, France \\ CNR, University of Salento, Italy \\ Ghent University/iMinds, Belgium \\ CSIRO, Australia \\ University of Antwerp, Belgium
}

\section{Organizing Committee}

Pier Luigi Carcagni

Marco Del Coco

Marco Leo

Pier Luigi Mazzeo

Paolo Spagnolo
Consiglio Nazionale delle Ricerche, Italy

CNR, Italy

Consiglio Nazionale delle Ricerche, Italy

Consiglio Nazionale delle Ricerche, Italy

Consiglio Nazionale delle Ricerche, Italy

\section{Program Committee}

Alin Achim
Sos Agaian
Hamid Aghajan
Edoardo Ardizzone
Atilla Baskurt
Sebastiano Battiato
Fabio Bellavia
Jenny Benois-Pineau
Bir Bhanu
Philippe Bolon
Egor Bondarev
Salah Bourennane
Catarina Brites
Alfred M. Bruckstein
Vittoria Bruni
Dumitru Dan Burdescu
Tiago Carvalho
Giuseppe Cattaneo
Andrea Cavallaro
Emre Celebi
Jocelyn Chanussot
Eric Debreuve

University of Bristol, UK

The University of Texas, USA

Stanford University, USA

University of Palermo, Italy

INSA, France

University of Catania, Italy

Università degli Studi di Palermo, Italy

Université Bordeaux 1, France

University of California, USA

University of Savoie, France

Technische Universiteit Eindhoven, The Netherlands

Ecole Centrale de Marseille, France

Instituto Superior Técnico, Portugal

Technion IIT, Israel

University of Rome La Sapienza, Italy

University of Craiova, Romania

Instituto Federal de São Paulo, Campinas, Brazil

University of Salerno, Italy

Queen Mary University of London, UK

Louisiana State University, USA

Université de Grenoble Alpes, France

CNRS, France 
Frédéric Dufaux

Jean-Luc Dugelay

Don Fraser

Toshiaki Fujii

Jérôme Gilles

Georgy Gimel'farb

Daniele Giusto

Bart Goossens

Lewis Griffin

David Helbert

Michael Hild

Mark Holden

Kazuhiro Hotta

Heikki Huttunen

Dimitris Iakovidis

Yuji Iwahori

Arto Kaarna

Zoltan Kato

Richard Kleihorst

Ludovic Macaire

Xavier Maldague

Gonzalo Pajares

Martinsanz

Takashi Matsuyama

Alfred Mertins

Jean Meunier

Massimo Minervini

Amar Mitiche

Adrian Munteanu

António J.R. Neves

Jennifer Newman

Nikos Paragios

Fernando

Pérez-González

Stuart Perry

Aleksandra Pizurica

Ljiljana Platisa

William Puech

Giovanni Ramponi

Dan Raviv

Paolo Remagnino

Patrice Rondao Alface

Florence Rossant

Luis Salgado Alvarez de Sotomayor

Carlo Sansone
ENST, France

EURECOM, France

University of New South Wales, Australia

Nagoya University, Japan

San Diego State University, USA

The University of Auckland, New Zealand

University of Cagliari, Italy

Ghent University/iMinds, Belgium

University College, UK

University of Poitiers, France

Osaka Electro-Communication University, Japan

Kyoto University, Japan

Meijo University, Japan

Tampere University of Technology, Finland

University of Thessaly, Greece

Chubu University, Japan

Lappeenranta University of Technology, Finland

University of Szeged, Hungary

Senso2Me and Ghent University, Belgium

Université Lille 1 Sciences et Technologies, France

Université Laval, Canada

Universidad Complutense, Spain

Graduate School of Informatics, Japan

Universität zu Lübeck, Germany

Université de Montréal, Canada

IMT School for Advanced Studies, Italy

INRS, Canada

Vrije Universiteit Brussel, Belgium

University of Aveiro, Portugal

Iowa State University, USA

Ecole Centrale de Paris, France

University of Vigo, Spain

Canon Information Systems Research Australia, Australia

Ghent University/iMinds, Belgium

Ghent University/iMinds, Belgium

LIRMM, France

University of Trieste, Italy

Massachusetts Institute of Technology, USA

Kingston University, UK

Nokia Bell Labs, Belgium

ISEP, France

Universidad Politécnica de Madrid, Spain

Università degli Studi di Napoli Federico II, Italy 


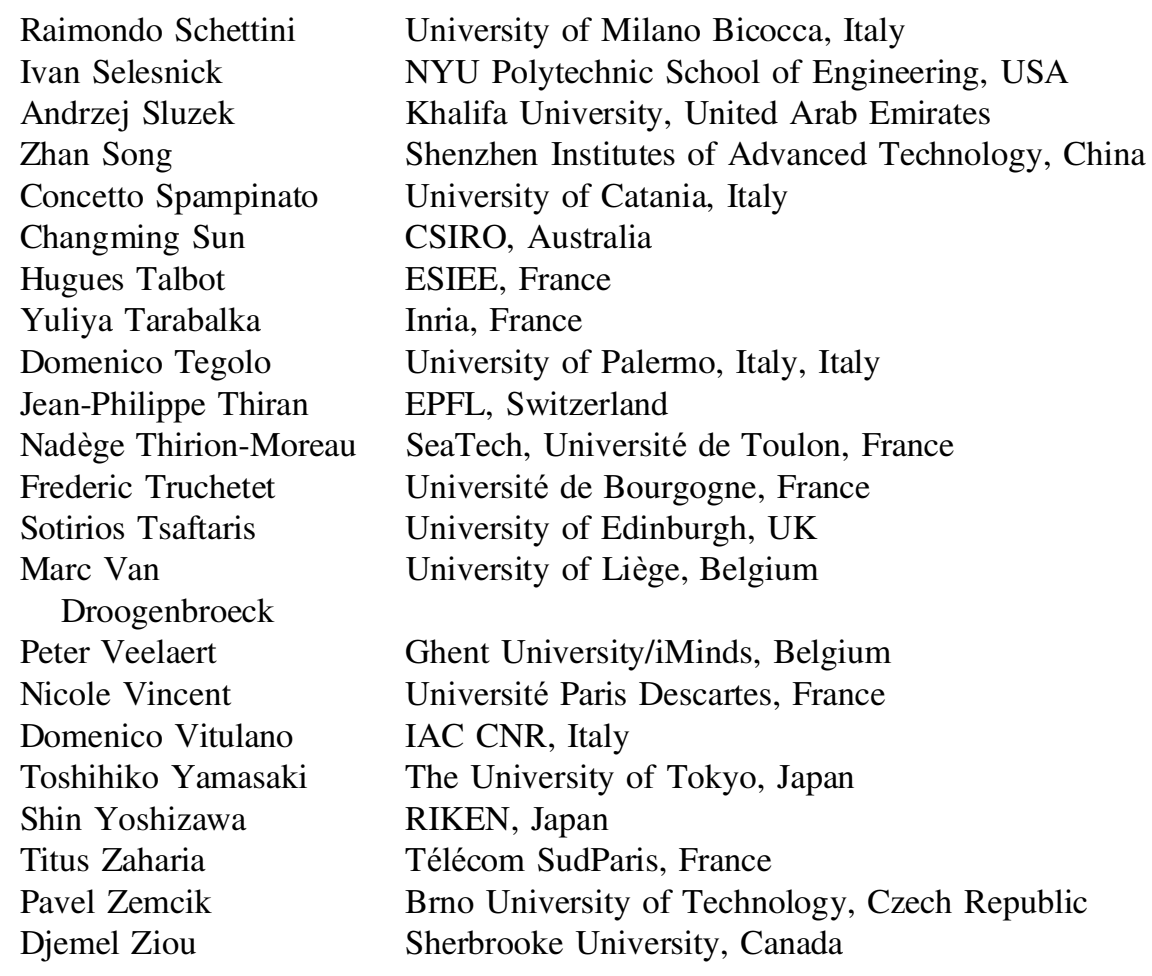

\section{Additional Reviewers}

Alin Achim

Jan Aelterman

Hamid Aghajan

Edoardo Ardizzone

Fabio Bellavia

Jenny Benois-Pineau

Jacques Blanc-Talon

Nyan Bo Bo

Philippe Bolon

Egor Bondarev

Salah Bourennane

Catarina Brites

Alfred M. Bruckstein

Vittoria Bruni

Dumitru Dan Burdescu

Pier Luigi Carcagni

Tiago Carvalho

Giuseppe Cattaneo

Jocelyn Chanussot

Francis Deboeverie
University of Bristol, UK

Ghent University, Belgium

Stanford University, USA

University of Palermo, Italy

Università degli Studi di Palermo, Italy

Université Bordeaux 1, France

DGA, France

Gent University/iMinds, Belgium

University of Savoie, France

Technische Universiteit Eindhoven, The Netherlands

Ecole Centrale de Marseille, France

Instituto Superior Técnico, Portugal

Technion IIT, Israel

University of Rome La Sapienza, Italy

University of Craiova, Romania

Consiglio Nazionale delle Ricerche, Italy

Instituto Federal de São Paulo, Campinas, Brazil

University of Salerno, Italy

Université de Grenoble Alpes, France

Ghent University, Belgium 


\begin{tabular}{|c|c|}
\hline Eric Debreuve & CNRS, France \\
\hline Marco Del Coco & CNR, Italy \\
\hline Cosimo Distante & CNR, University of Salento, Italy \\
\hline Simon Donné & iMinds-IPI-UGent, Belgium \\
\hline Martin Drahansky & Czech Republic \\
\hline Frédéric Dufaux & ENST, France \\
\hline Jean-Luc Dugelay & EURECOM, France \\
\hline Mohamed Eldib & UGent-IPI-IMinds, Belgium \\
\hline $\begin{array}{l}\text { Giovanni Maria } \\
\text { Farinella }\end{array}$ & University of Catania, Italy \\
\hline Don Fraser & University of New South Wales, Australia \\
\hline Michal Fularz & Poznan University of Technology, Poland \\
\hline Jérôme Gilles & San Diego State University, USA \\
\hline Georgy Gimel'farb & The University of Auckland, New Zealand \\
\hline Daniele Giusto & University of Cagliari, Italy \\
\hline Bart Goossens & Ghent University/iMinds, Belgium \\
\hline Junzhi Guan & Ghent University, Belgium \\
\hline Monson Hayes & Georgia Institute of Technology, USA \\
\hline David Helbert & University of Poitiers, France \\
\hline Michael Hild & Osaka Electro-Communication University, Japan \\
\hline Mark Holden & Kyoto University, Japan \\
\hline Kazuhiro Hotta & Meijo University, Japan \\
\hline Heikki Huttunen & Tampere University of Technology, Finland \\
\hline Dimitris Iakovidis & University of Thessaly, Greece \\
\hline Yuji Iwahori & Chubu University, Japan \\
\hline Ljubomir Jovanov & Ghent University/iMinds, Belgium \\
\hline Arto Kaarna & Lappeenranta University of Technology, Finland \\
\hline Marek Kraft & Poznan University of Technology, Poland \\
\hline Simon Lacroix & LAAS, France \\
\hline Marco Leo & National Research Council of Italy, Italia \\
\hline Wenzhi Liao & Gent University, Belgium \\
\hline Ludovic Macaire & Université Lille 1 Sciences et Technologies, France \\
\hline $\begin{array}{c}\text { Gonzalo Pajares } \\
\text { Martinsanz }\end{array}$ & Universidad Complutense, Spain \\
\hline Pier Luigi Mazzeo & Consiglio Nazionale delle Ricerche, Italy \\
\hline Massimo Minervini & IMT School for Advanced Studies, Italy \\
\hline Amar Mitiche & INRS, Canada \\
\hline Adrian Munteanu & Vrije Universiteit Brussel, Belgium \\
\hline António J.R. Neves & University of Aveiro, Portugal \\
\hline Jennifer Newman & Iowa State University, USA \\
\hline Benhur Ortiz & Ghent University, Belgium \\
\hline Rudi Penne & Anwerp University, Belgium \\
\hline $\begin{array}{l}\text { Fernando } \\
\text { Pérez-González }\end{array}$ & University of Vigo, Spain \\
\hline Wilfried Philips & Ghent University/iMinds, Belgium \\
\hline Aleksandra Pizurica & Ghent University/iMinds, Belgium \\
\hline
\end{tabular}




Ljiljana Platisa
Dan Popescu
Dan Popescu
Giovanni Ramponi
Patrice Rondao Alface
Florence Rossant
Luis Salgado Alvarez de
Sotomayor
Carlo Sansone
Raimondo Schettini
Paul Scheunders
Adam Schmidt
Ivan Selesnick
Vasileios Sevetlidis
Andrzej Sluzek
Paolo Spagnolo
Changming Sun
Hugues Talbot
Yuliya Tarabalka
Nadège Thirion-Moreau
Frederic Truchetet
Marc Van
Droogenbroeck
Peter Veelaert
Nicole Vincent
Domenico Vitulano
Michiel Vlaminck
Xingzhe Xie
Shin Yoshizawa
Pavel Zemcik
Djemel Ziou
Witold Zorski
Pariong

Ghent University/iMinds, Belgium

University Politehnica of Bucharest, Romania

CSIRO, Australia

University of Trieste, Italy

Nokia Bell Labs, Belgium

ISEP, France

Universidad Politécnica de Madrid, Spain

Università degli Studi di Napoli Federico II, Italy

University of Milano Bicocca, Italy

University of Antwerp, Belgium

University of Rouen, France

NYU Polytechnic School of Engineering, USA

University of Edinburgh, UK

Khalifa University, United Arab Emirates

National Research Council, Italy

CSIRO, Australia

ESIEE, France

Inria, France

SeaTech, Université de Toulon, France

Université de Bourgogne, France

University of Liège, Belgium

Ghent University/iMinds, Belgium

Université Paris Descartes, France

IAC CNR, Italy

Ghent University, Belgium

Ghent University, Belgium

RIKEN, Japan

Brno University of Technology, Czech Republic

Sherbrooke University, Canada

Military University of Technology, Poland 


\section{Contents}

Gradients versus Grey Values for Sparse Image Reconstruction

and Inpainting-Based Compression . . . . . . . . . . . . . . . . . 1

Markus Schneider, Pascal Peter, Sebastian Hoffmann,

Joachim Weickert, and Enric Meinhardt-Llopis

Global Bilateral Symmetry Detection Using Multiscale Mirror Histograms . . .

Mohamed Elawady, Cécile Barat, Christophe Ducottet,

and Philippe Colantoni

Neural Network Boundary Detection for 3D Vessel Segmentation. . . . . . . .

Robert Ieuan Palmer and Xianghua Xie

A Simple Human Activity Recognition Technique Using DCT . . . . . . . . . Aziz Khelalef, Fakhreddine Ababsa, and Nabil Benoudjit

Hand Gesture Recognition Using Infrared Imagery Provided

by Leap Motion Controller. . . . . . . . . . . . . . . . . . . .

Tomás Mantecón, Carlos R. del-Blanco, Fernando Jaureguizar, and Narciso García

Horizon Line Detection from Fisheye Images Using Color Local Image

Region Descriptors and Bhattacharyya Coefficient-Based Distance . . . . . . .

Youssef El merabet, Yassine Ruichek, Saman Ghaffarian, Zineb Samir,

Tarik Boujiha, Raja Touahni, and Rochdi Messoussi

Joint Segmentation of Myocardium on Rest and Stress Spect Images. . . . . .

Marc Filippi, Michel Desvignes, Anastasia Bozok,

Gilles Barone-Rochette, Daniel Fagret, Laurent Riou,

and Catherine Ghezzi

Parallel Hough Space Image Generation Method for Real-Time

Lane Detection . . . . . . . . . . . . . . . . . . . . . .

Hee-Soo Kim, Seung-Hae Beak, and Soon-Yong Park

A Novel Decentralised System Architecture for Multi-camera

Target Tracking . .........................

Gaetano Di Caterina, Trushali Doshi, John J. Soraghan,

and Lykourgos Petropoulakis

Intramolecular FRET Efficiency Measures for Time-Lapse Fluorescence

Microscopy Images $\ldots \ldots \ldots \ldots \ldots \ldots \ldots \ldots \ldots \ldots \ldots \ldots \ldots \ldots$ Mark Holden 
Predicting Image Aesthetics with Deep Learning. . . . . . . . . . . . .

Simone Bianco, Luigi Celona, Paolo Napoletano,

and Raimondo Schettini

Automatic Image Splicing Detection Based on Noise Density Analysis

in Raw Images . . . . . . . . . . . . . . . . . . . . . .

Thibault Julliand, Vincent Nozick, and Hugues Talbot

Breast Shape Parametrization Through Planar Projections. . . . . . . . . .

Giovanni Gallo, Dario Allegra, Yaser Gholizade Atani,

Filippo L.M. Milotta, Filippo Stanco, and Giuseppe Catanuto

Decreasing Time Consumption of Microscopy Image Segmentation

Through Parallel Processing on the GPU . . . . . . . . . . . . .

Joris Roels, Jonas De Vylder, Yvan Saeys, Bart Goossens,

and Wilfried Philips

Coral Reef Fish Detection and Recognition in Underwater Videos by Supervised Machine Learning: Comparison Between Deep Learning

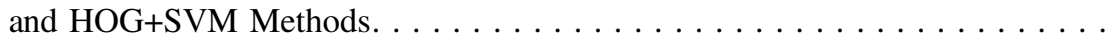

Sébastien Villon, Marc Chaumont, Gérard Subsol, Sébastien Villéger,

Thomas Claverie, and David Mouillot

A Real-Time Eye Gesture Recognition System Based on Fuzzy Inference System for Mobile Devices Monitoring . . . . . . . . . . . . . . .

Hanene Elleuch, Ali Wali, Anis Samet, and Adel M. Alimi

Spatially Varying Weighting Function-Based Global and Local Statistical Active Contours. Application to X-Ray Images. . . . . . . . . . . . . . Aicha Baya Goumeidane and Nafaa Nacereddine

Vegetation Segmentation in Cornfield Images Using Bag of Words. . . . . . . Yerania Campos, Erik Rodner, Joachim Denzler, Humberto Sossa, and Gonzalo Pajares

Fast Traffic Sign Recognition Using Color Segmentation and Deep Convolutional Networks. . . . . . . . . . . . . . . . . . . Ali Youssef, Dario Albani, Daniele Nardi, and Domenico Daniele Bloisi

The Orlando Project: A 28 nm FD-SOI Low Memory Embedded Neural Network ASIC . . . . . . . . . . . . . . . . . . . . . . . Giuseppe Desoli, Valeria Tomaselli, Emanuele Plebani, Giulio Urlini, Danilo Pau, Viviana D'Alto, Tommaso Majo, Fabio De Ambroggi, Thomas Boesch, Surinder-pal Singh, Elio Guidetti, and Nitin Chawla

Factor Analysis of Dynamic Sequence with Spatial Prior for 2D Cardiac Spect Sequences Analysis . . . . . . . . . . . . . . . . . . . Marc Filippi, Michel Desvignes, Eric Moisan, Catherine Ghezzi, Pascale Perret, and Daniel Fagret 
Soccer Player Detection with only Color Features Selected Using Informed

Haar-like Features . . . . . . . . . . . . . . . . . . . . . . . . . . . . . .

Ryusuke Miyamoto and Takuro Oki

Person Re-identification in Frontal Gait Sequences via Histogram

of Optic Flow Energy Image . . . . . . . . . . . . . . . . . . . . .

Athira Nambiar, Jacinto C. Nascimento, Alexandre Bernardino,

and José Santos-Victor

A Bayesian Approach to Linear Unmixing in the Presence of Highly

Mixed Spectra . . . . . . . . . . . . . . . . . . . .

Bruno Figliuzzi, Santiago Velasco-Forero, Michel Bilodeau, and Jesus Angulo

Key Frames Extraction Based on Local Features for Efficient

Video Summarization . . . . . . . . . . . . . . . . . . . . .

Hana Gharbi, Mohamed Massaoudi, Sahbi Bahroun,

and Ezzeddine Zagrouba

A Simple Evaluation Procedure for Range Camera Measurement Quality. . . .

Boris Bogaerts, Rudi Penne, Seppe Sels, Bart Ribbens,

and Steve Vanlanduit

Accordion Representation Based Multi-scale Covariance Descriptor

for Multi-shot Person Re-identification. . . . . . . . . . . . . . . . . . .

Bassem Hadjkacem, Walid Ayedi, and Mohamed Abid

Jensen Shannon Divergence as Reduced Reference Measure

for Image Denoising . . . . . . . . . . . . . . . . . . . .

Vittoria Bruni and Domenico Vitulano

Visual Localization Using Sequence Matching Based on Multi-feature

Combination. . . . . . . . . . . . . . . . . . . . . . . . . .

Yongliang Qiao, Cindy Cappelle, and Yassine Ruichek

Towards Automated Drone Surveillance in Railways: State-of-the-Art

and Future Directions . . . . . . . . . . . . . . . . . .

Francesco Flammini, Riccardo Naddei, Concetta Pragliola, and Giovanni Smarra

Combining Stacked Denoising Autoencoders and Random Forests

for Face Detection. . . . . . . . . . . . . . . . . . . . . . . .

Jingjing Deng, Xianghua Xie, and Michael Edwards

Multimodal Registration of PET/MR Brain Images Based

on Adaptive Mutual Information . . . . . . . . . . . . . . . . . . .

Abir Baâzaoui, Mouna Berrabah, Walid Barhoumi,

and Ezzeddine Zagrouba 
Aerial Detection in Maritime Scenarios Using Convolutional Neural

Networks . . . . . . . . . . . . . . . . . . . . . . . . . . . . . . . . .

Gonçalo Cruz and Alexandre Bernardino

$\mathrm{R}^{3} \mathrm{P}$ : Real-time RGB-D Registration Pipeline . . . . . . . . . . . .

Hani Javan Hemmat, Egor Bondarev, and Peter H.N. de With

Vector Quantization Enhancement for Computer Vision Tasks . . . . . . . . . . Remi Trichet and Noel E. O'Connor

Learning Approaches for Parking Lots Classification. . . . . . . . . . . . . . Daniele Di Mauro, Sebastiano Battiato, Giuseppe Patanè, Marco Leotta, Daniele Maio, and Giovanni M. Farinella

Video Event Detection Based Non-stationary Bayesian Networks . . . . . . . . . Christophe Gonzales, Rim Romdhane, and Séverine Dubuisson

Optimized Connected Components Labeling with Pixel Prediction . . . . . . . . Costantino Grana, Lorenzo Baraldi, and Federico Bolelli

Hierarchical Fast Mean-Shift Segmentation in Depth Images . . . . . . . . . . . Milan Šurkala, Radovan Fusek, Michael Holuša, and Eduard Sojka

Robust Color Watermarking Method Based on Clifford Transform . . . . . . . . Maroua Affes, Malek Sellami Meziou, and Faouzi Ghorbel

Action-02MCF: A Robust Space-Time Correlation Filter for Action Recognition in Clutter and Adverse Lighting Conditions . . . . . . . . . . . . Anwaar Ulhaq, Xiaoxia Yin, Yunchan Zhang, and Iqbal Gondal

An Image Quality Metric with Reference for Multiply Distorted Image . . . . . Aladine Chetouani

3D Planar RGB-D SLAM System. . . . . . . . . . . . . . . . . . . . Hakim ElChaoui ElGhor, David Roussel, Fakhreddine Ababsa, and El-Houssine Bouyakhf

Towards a Generic M-SVM Parameters Estimation Using Overlapping Swarm Intelligence for Handwritten Characters Recognition . . . . . . . . . . . . Marwa Amara, Kamel Zidi, and Khaled Ghedira

Human Action Recognition Based on Temporal Pyramid of Key

Poses Using RGB-D Sensors . . . . . . . . . . . . . . . . . . . . . . .

Enea Cippitelli, Ennio Gambi, Susanna Spinsante, and Francisco Florez-Revuelta

Multi-layer Dictionary Learning for Image Classification . . . . . . . . . . . 
Intelligent Vision System for ASD Diagnosis and Assessment . . . . . . . . . .

Marco Leo, Marco Del Coco, Pierluigi Carcagni, Pier Luigi Mazzeo,

Paolo Spagnolo, and Cosimo Distante

Visual Target Detection and Tracking in UAV EO/IR Videos by Moving

Background Subtraction . . . . . . . . . . . . . . . . . .

Francesco Tufano, Cesario Vincenzo Angelino, and Luca Cicala

A Multiphase Level Set Method on Graphs for Hyperspectral Image

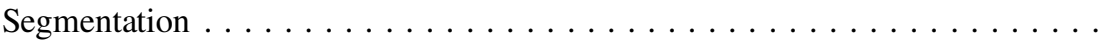

Kaouther Tabia, Xavier Desquesnes, Yves Lucas, and Sylvie Treuillet

A Mobile Application for Leaf Detection in Complex Background

Using Saliency Maps. . . . . . . . . . . . . . . . . . . . . .

Lorenzo Putzu, Cecilia Di Ruberto, and Gianni Fenu

Content-Based Mammogram Retrieval Using Mixed Kernel PCA

and Curvelet Transform . . . . . . . . . . . . . . . . . . . . . . .

Sami Dhahbi, Walid Barhoumi, and Ezzeddine Zagrouba

Combination of RGB-D Features for Head and Upper Body

Orientation Classification . . . . . . . . . . . . . . . . . . . . . . . . . .

Laurent Fitte-Duval, Alhayat Ali Mekonnen, and Frédéric Lerasle

A Parametric Algorithm for Skyline Extraction . . . . . . . . . . . . . . Mehdi Ayadi, Loreta Suta, Mihaela Scuturici, Serge Miguet, and Chokri Ben Amar

Quaternion Linear Color Edge-Glowing Filter Using Genetic Algorithm . . . . Shagufta Yasmin and Stephen J. Sangwine

Scalable Vision System for Mouse Homecage Ethology. . . . . . . . . . . . . . . Ghadi Salem, Jonathan Krynitsky, Brett Kirkland, Eugene Lin, Aaron Chan, Simeon Anfinrud, Sarah Anderson, Marcial Garmendia-Cedillos, Rhamy Belayachi, Juan Alonso-Cruz, Joshua Yu, Anthony Iano-Fletcher, George Dold, Tom Talbot, Alexxai V. Kravitz, James B. Mitchell, Guanhang Wu, John U. Dennis, Monson Hayes, Kristin Branson, and Thomas Pohida

Spatiotemporal Features Learning with 3 DPyraNet $\ldots \ldots \ldots \ldots \ldots$ Ihsan Ullah and Alfredo Petrosino

Automatic Segmentation of TV News into Stories Using Visual and Temporal Information $\ldots \ldots \ldots \ldots \ldots \ldots \ldots$ Bogdan Mocanu, Ruxandra Tapu, and Titus Zaharia

Wavelet Neural Network Initialization Using LTS for DNA Sequence Classification . . . . . . . . . . . . . . . . . . . . . . . . . . 
Collection of Visual Data in Climbing Experiments for Addressing the Role of Multi-modal Exploration in Motor Learning Efficiency . . . . . . . .

Adam Schmidt, Dominic Orth, and Ludovic Seifert

Fog Augmentation of Road Images for Performance Analysis of Traffic

Sign Detection Algorithms . . . . . . . . . . . . . . . . .

Thomas Wiesemann and Xiaoyi Jiang

Statistical Modeling Based Adaptive Parameter Setting for Random

Walk Segmentation . . . . . . . . . . . . . . . . . . . . . .

Ang Bian and Xiaoyi Jiang

On-the-Fly Architecture Design and Implementation of a Real-Time

Stereovision System . . . . . . . . . . . . . . . . . . . . . Mohamed B.M. Masmoudi, Chadlia Jerad, and Rabah Attia

Complex Image Processing Using Correlated Color Information . . . . . . . . . . Dan Popescu, Loretta Ichim, Diana Gornea, and Florin Stoican

Using PNU-Based Techniques to Detect Alien Frames in Videos . . . . . . . 735 Giuseppe Cattaneo, Gianluca Roscigno, and Andrea Bruno

Author Index 